\title{
Improvement of Surface Finish by Multiple Piezoelectric Transducers in Fused Deposition Modelling
}

\author{
A. S. Mohamed", S. Maidin", S. B. Mohamed", M. K. Muhamad*, J. H. U. Wong*, W. F. A. Romlee* \\ Faculty of Manufacturing Engineering, Universiti Teknikal Malaysia Melaka, Melaka, 76100, Malaysia \# \\ E-mail: asyafiqmohamed@gmail.com, shajahan@utem.edu.my \\ * Faculty of Innovative Design and Technology, Universiti Sultan Zainal Abidin,Terengganu, 21300, Malaysia y \\ E-mail: saifulbahri@unisza.edu.my
}

\begin{abstract}
Additive Manufacturing (AM) which embrace as a new range technology of creating and producing end user parts in term of adding material layer by layer to create solid objects from 3D CAD data. AM in particular Fused Deposition Modelling (FDM) used (ABS) thermoplastic have shown the most popular among the industry as its technology can print complex geometrical part without human intervention and tools. However, FDM fierce enemy whereas the common problem of stair-stepping, which means that seam lines appear between layers and excess material if often left as a residue, cause to lead rough surface and poor quality finish. It is often desirable for an AM model to have aesthetic or functional importance. Hence, reducing layer thickness will generally improve surface roughness but will add to the build time for the model. As an interest investigates the use of ultrasonic for FDM, this experiment will focus on the effect of applying multiple piezoelectric transducer for FDM printer. This paper aims to explore the effect use of multiple piezoelectric with different frequency applied $(27,40,50 \mathrm{kHz})$ to improve surface finish quality part printed by FDM whereby an ultrasonic transducer firmly attached onto the platform. Optical microscope with the aid of pro VIS software version 2.90 was used to measure the quality of surface roughness of samples printed with vibration in the above stated frequency. Hence, it was found that 1 piezo with $50 \mathrm{kHz}$ frequency applied to the FDM machine achieved improve surface finish due to less layer thickness defect and finer layer thickness produce.
\end{abstract}

Keywords - additive manufacturing, fused deposition modelling, ultrasonic frequency, surface finish.

\section{INTRODUCTION}

Additive Manufacturing (AM) technologies have come through an evolution of Rapid Prototyping (RP) technologies over the last decade in the industry. AM which embrace as a new range technology of creating and producing end-user parts accurately in term of adding or building up material layer by layer to create an object from 3D CAD data without human intervention [1], [2]. The basic principle of this technology is that the drawing initially produced using three dimensional Computer Aided Design (3D CAD) system and fabricated directly from CAD data without process planning [3]. Nowadays, AM technologies allow us to fabricate product that in high added value and this process called as "clean processes as this process only apply the precise amount of stuff. In summation, the energy consumption also less and limited compared with the machining procedure. In fact, AM is process which can instantly obtain functional part from CAD model with only one manufacturing step. Meanwhile, the machining process needs to consider few step of manufacturing process to complete the fabrication of a product [4].

As it become more widely available, AM in particular Fused Deposition Modeling (FDM) system that use Acrylonitrile Butadiene Styrene (ABS) thermoplastic have shown to be the most known and popular among the industry as the technology can printed complex geometrical part without using tools and human intervention [5]. In FDM process, build material in the form of low viscosity and flexible filament is partially melted and extruded throughout from a robotically controlled hot end deposition nozzle onto a table which called hot bed in a temperature controlled environment for printed the 3D part layer by layer. The 3D part takes the form of laminate composite with vertically stacked layers consisting of contiguous material fibre (raster) with interstitial voids (air gap). The bonding between neighbouring fibres takes place via thermally driven diffusion welding [6]. Nevertheless, despite its capability to build functional part with complex geometrical shapes, most molten layered thermoplastic surface often unlevel which lead towards rough and poor. 
Conversely, ultrasound is a sound that frequencies greater than $20 \mathrm{kHz}$ (ANSI). Ultrasound is a proven technology that has been extensively used for machining and it has been claimed to improve surface quality for work piece [7]. The use of ultrasonic vibration in different manufacturing processes well documented for more than 50 years [8]. The purpose of this research is to investigate and study the novel use of ultrasonic assisted approach to enhance the surface quality. Literature review has found that there is a gap in knowledge, where ultrasound only applied in subtractive machining and little work has been done to study the application of ultrasound for AM. Based on the previous paper, it is found that by addapting pieozelectric will improve surface roughness. However, using multiple piezoelectric transducer never been done before. As an interest to study and investigate the use of ultrasonic for FDM, this study will focus on the effect of applying multiple piezoelectric transducer for FDM printer. This paper aims to explore the use of multiple piezoelectric to improve surface finish quality printed part by a destop FDM machine.

\section{A. Fused Deposition Modelling}

FDM is a technology called Fused Deposition Modeling that in the group of extrusion based process in AM system which is develop by S. Scott Crump in the late year of 1980s, until then it been commercialized by Stratsys in the year 1990s [9]. In the production of 3D solid complex model from the CAD data without the aid of tools and human intervention, FDM is a good choice of AM technology to be used [5]. FDM machine is an additive processes which use thermoplastic filaments to print polymer parts from 3DCAD data that is sliced into layers and converted to STL file type. Thus, the file will be transferred into FDM machine for tool path generation and support structure calculation. The support and build materials are a continuous filament held on a spool that fed through heated hot end nozzle. The material particularly heated to semi-liquid state, which reduce the viscosity, then will be extruded using robotic nozzle which is controlled by $\mathrm{x}, \mathrm{y}$ and $\mathrm{z}$ axis movement to form of layer by layer printing process. The thermal fusion generally will allow the molten polymer layered in between to bond by one another and solidify [1], [9]-[13]. For each layer, the process is repeated until a finish product is printed. Throughout theimplementation of FDM, various application can be produced such as functional testing, design verification and last but not least design study [14]. Moreover, FDM enable swift development process, outstandingly handling multiplex geometries quickly and rapidly unlike other manufacturing [11]. FDM methods offer tremendous benefits that meet an ideal prototype machine such as lesser build time, cost effective and elimination of expensive flexibility and tooling which caused it involve in most of the industrial field. Furthermore, FDM will have advantages in time consumption and cost investment which is more economically compared to conventional manufacturing [10]. However, FDM fierce enemy whereas the common problem of stair-stepping which means that seam lines appear between layers and excess material if often left as a residue, cause to lead rough surface and poor quality finish. It is often desirable for an AM model to have aesthetic or functional importance. Hence, reducing layer thickness will generally improve surface roughness but will add to the build time for the model [15], [16]. To eliminate and improve this, numerous researchers have suggest to achieve better surface finish by (i) post processing using chemical treatment (90\% dimethyketone and $10 \%$ water) as a post processing process to FDM parts tremendously shown prospective potential, but at the expenses of a negligible change in the prototype [17] (ii) slicing strategy which a new approach to enhanced surface roughness by using a theoretical model to represent surface roughness circulation based on different surface angle and as a main factor that significantly affect surface roughness [18] (iii) optimal build orientation which most quality possible surface finish on FDM can be archive by selecting the optimal FDM process parameters such build orientation and interaction of these parameter and considering thickness of layers [19] (iv) fabrication parameter optimization such as the principle error of RP process, the inherent characteristic of FDM and some micro-scratches on the surface of the extruded material [20]. Accoding to [21], it pointed out that the most quality possible surface finish on FDM can be archive by selecting the optimal FDM process parameters such build orientation and interaction of these parameter and considering thickness of layers. Similarly, in [22] regulate experimental study to examine surface roughness value as a function of build orientation, road width, layer thickness and raster angle for FDM printed part. Last but not least, in [23] expressed the opinion that using chemical treatment to FDM parts tremendously shown prospective potential, but at the expenses of a negligible change in the prototype. Despite this, most of these technique are ineffective due to the requirements of further processes to improve FDM printed parts surface quality which in demand of time and cost consuming.

\section{B. Ultrasound}

In [24] presented a comprehensive review of ultrasonicassisted machining. The paper showed that ultrasonic machining has been principallypracticed on brittle materials. Although removal rates are not high, ultrasonic technology applicable very well this type of material. Ultrasonic assisted machining has been proven to be an efficient technique for improving the machinability of several aeronautic materials such as aluminium [24] or Inconel 718 [25]. The process parameters studied assisted with vibration application in conventional machine cutting processes are such as chips breaking, burr generation, work piece roughness, tool life or torque and cutting forces are some [26]. Ultrasonic assisted machining process is non-thermal, non-chemical and does not require the work piece to be electrically conducted. Due to that, there are no adverse integrity effects thus generating compressive residual stresses on the work piece that consequentially promotes improved fatigue strength. Ultrasound is a technology that is proven to be used thoroughly for machining and also been claimed to enhance surface quality of the work piece [27]. The piezoelectric components creates ultrasonic vibration which vibrate in vertical direction happen to be used for assisting laser machining and the outcome of the process produced a superior quality of surface finish [28]. The process of ultrasonic assisted machining does not require the work 
piece become electrically conducted because it is nonthermal and non-chemical process. As an outcome, there are no negative side effects and yet it increased fatigue strength. In addition, this research suggests the utilization of ultrasound by transforming low frequency electrical signal $(60 \mathrm{~Hz})$ to a high-frequency electrical signal (approximately $20 \mathrm{kHz}$ ) which is supplied to a transducer. In [29] identifiesthe usage of ultrasound is increasingly applied in various industrial applications, which the technology is proven to be able to improve the quality of machined surface finish. The tool vibrates at a high regularity frequently higher than $20 \mathrm{kHz}$ and abrasive slurry is pumped between the work piece as well as the tool in ultrasonic machining [30]. The process which involved usage of ultrasonic is considered safe because it does not create a chemical reaction and does not chemically corrode the work piece. Ultrasonic vibration is a practical method to unleash the local energy concentration because a high frequency repetitive motion has an effect normalizing the spatially concentrated energy uniformly. In current decades, a number of researchers have investigated the impact of ultrasonic vibration in machining with the aim to enhance the quality of machined surfaces. The majority of the ultrasonic research was conducted in the discipline of subtractive manufacturing and the information of its application in the AM, especially FDM very limited. In [24] has performed experimental investigations on the impact of ultrasonic vibration in nanosecond laser machining on the workpiece surface finish. In [31] have summarized that the ultrasonic assisted machining is an advanced processing technology has a potential to enhance the machining procedure, especially for stiff material. In [32] researched on the ultrasonic additive manufacturing in a hybrid production process and discovered the processes of adding ultrasonic are matched to high tech metal matrix composites with high temperatures and pressure. In [33] studied the effect of ultrasonic on grinding of the Ti6A14v alloy by using an ultrasonic frequency range of $20 \mathrm{kHz}$ that applied to the work piece and discovered a decrease of grinding forces and positive enhancement on surface roughness. In [28] has performed experimental investigations by using piezoelectric transducers with $23.56 \mathrm{kHz}$ frequency to obtain and improve surface fabrication in order to overcome the micro sized holes on the surface of the workpiece that lack of high aspect ratio or a good surface finish. In [34] has summarized that the ultrasonic assisted micro-milling frequency is not based on high numerical value will give better results because in high frequency, the vibration will impact the cutter teeth tool life. Thus, it can be concluded that the quality of the processed will be based on ultrasonic frequency. To the best of our knowledge, many research works and published insight related to the improvement of AM part's surface finish only centered towards post processing activities which are parts build orientation, slicing strategy, chemical treatment and process parameter optimization. Nonetheless, no data have been published on the ultrasonic assisted FDM to produce a better surface finish. For that reason, a research on the ultrasonic assisted FDM including the comprehensive process parameter that related to the experiment is crucial to ensure there is improvement on productivity and surface quality of FDM parts.

\section{METHODOLOGY}

\section{A. Experimental Setup}

Fig. 1 presents UP Plus 2 FDM 3D printer which its printing size of $140 \mathrm{~mm} \times 140 \mathrm{~mm} \times 135 \mathrm{~mm}$ with a $0.04 \mathrm{~mm}$ nozzle diameter used to print sample. This is due to its capability to print outstanding build parameters through its thin layer thickness and road width, as well as being reconcilable with ultrasonic-assisted structure. Hence, the $3 \mathrm{D}$ printer was chosen by virtue of its acceptance among users in conjunction with its availability. The material that was proposed and used in this research is Acrylonitrile Butadiene Styrene (ABS) thermoplastic, which is widely used and familiar material for FDM machine. In order to assist the experiment, a common piezoelectric transducer performing in a horizontal wave or vibration mode was fixed and securely attached in contact with the hotbed of FDM machine. To guarantee the vibration will disseminate thoroughly, the piezoelectric transducer was attached to the whole surface. Most of the major challenge was the positioning of the piezoelectric transducer on the hotbed platform. The intention to assure that it mounted perfectly without making any contact or hitting 3D printer parts, while the $3 \mathrm{D}$ printer performing calibration and printing movement.

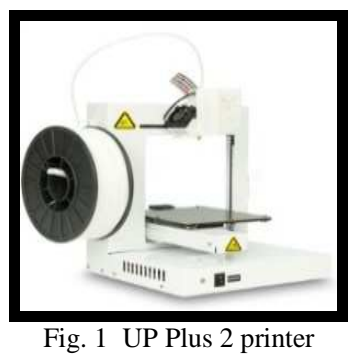

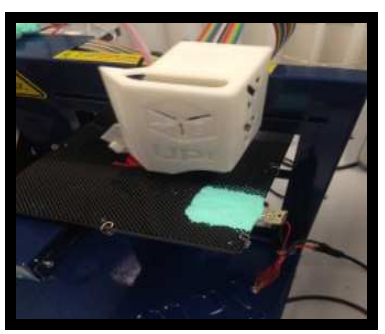

Fig. 2a 1 piezoelectric

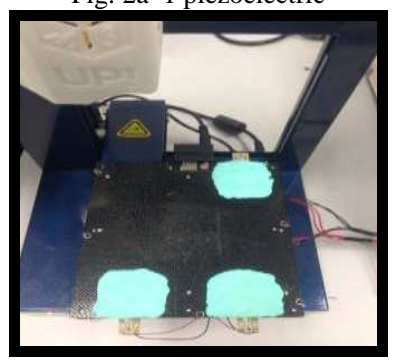

Fig. 2c 3 piezoelectrics



Fig. 2b 2 piezoelectrics

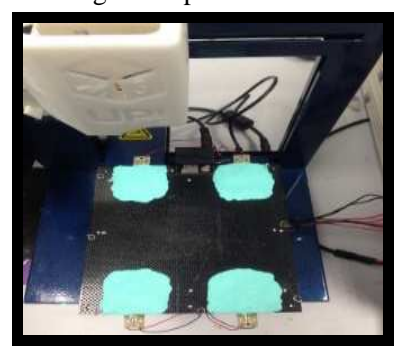

Fig. 2d 4 piezoelectrics
The positioning of the piezoelectric is due to the limitation and constrain of the 3D printer itself. In advance, by changing the position to a horizontal position, the piezoelectric by any chance will make contact and hit any parts of the 3D printer while printing, it will stop the printing and maybe cause damage or failure to function. There are four patterns of position for piezoelectric transducer as 
illustrated in Fig. 2a until $2 d$ to indicate the number of piezoelectric transducers.

A function generator with a maximum power of $20 \mathrm{~V}$ comes with an alterable frequency was used to supply electric power to the piezoelectric transducer. This experiment conducted with the frequency set at $27 \mathrm{kHz}$, which is an optimal frequency after performing Design of Experiment, while $40 \mathrm{kHz}$ and $50 \mathrm{kHz}$ appropriately was chosen randomly to study the effect of high frequency piezoelectric appliance on the sample with regards to the surface roughness quality. For each number of piezoelectric transducers used, one sample printed using the FDM UP Plus 2 3D printer. Fig. 3 shows a printed sample with square shape size of $2 \times 2 \times 2 \mathrm{~cm}$.

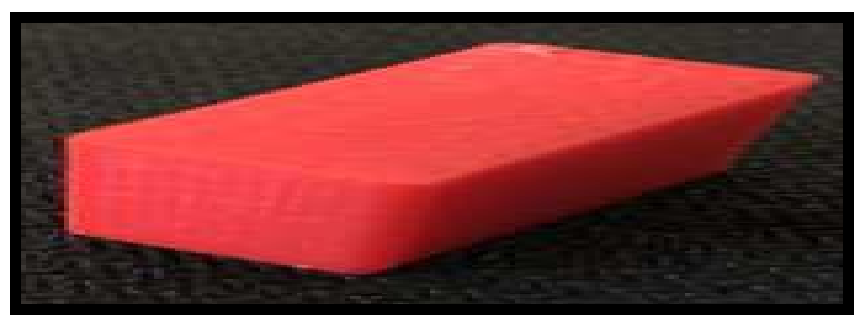

Fig. 3 Printed sample

\section{B. Evaluation Parameters}

Table I provides the FDM deposition parameters. For this experiment, the layer thickness, frequency and fill of surface were selected as influence factor that would affect the surface finish quality. The layer thickness and fill of surface parameters was set up based on the UP Plus 2 3D printer software and the frequency value set by the function generator. The layer thickness can be set from $0.2 \mathrm{~mm}$ per layer to $0.4 \mathrm{~mm}$ per layer. The finer layer thickness produced better quality, stronger the printed part and longer it takes to print. There are four types of honeycomb fill that could be selected from the 3D printer setting. However, only one type was choosing namely semi-solid honeycomb (level 1) due to time consideration, quality and better surface of model produced. In this research, the ultrasonic actuator will be vibrated by ultrasonic power supply with $27 \mathrm{kHz}, 40 \mathrm{kHz}$ and $50 \mathrm{kHz}$ and amplitude of $10 \mu \mathrm{m}$ which is the standard frequency.

TABLE I

PRINTING PARAMETERS

\begin{tabular}{|c|c|}
\hline Parameter & Value \\
\hline Layer thickness, $\mathrm{t}$ & $0.2 \mathrm{~mm}$ \\
\hline Frequency, $\mathrm{F}$ & $27 \mathrm{kHz}, 40 \mathrm{kHz}, 50 \mathrm{kHz}$ \\
\hline Fill of surface & Level 1 \\
\hline
\end{tabular}

The sample was drawn with CAD software. It was printed using the UP Plus 2 3D printer with a piezoelectric transducer assisted by attaching onto the built hotbed platform. With an aid of optical microscope with pro VIS software was used to capture images and evaluate the quality of surface roughness of the models printed with the vibration specified frequency is visible in Fig. 4.

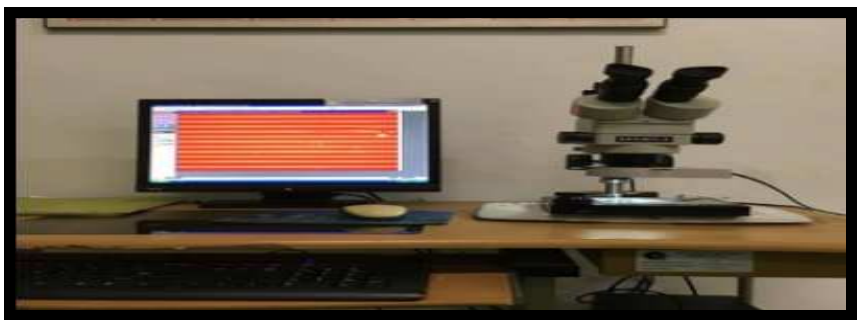

Fig. 4 Optical microscope to evaluate the surface roughness

\section{RESULTS AND DISCUSSION}

The experiment only focused on one critical surface whereby each model has 4 surfaces. Fig. 4(a-c) shows the critical surface selected to encompass the high defect and rough surfaces that viewed through an optical microscope. The consequence of frequency applied and the characterization of the surface roughness of each model as exhibits in Fig. 5. Surface roughness have been analyzed with the Portable Surface Roughness Tester Mitutoyo SJ301. The method of data construct is Ra, which is the arithmetic mean of the departures of the roughness profile from the mean line within the evaluation length. The data consists of 15 readings for every surface point.
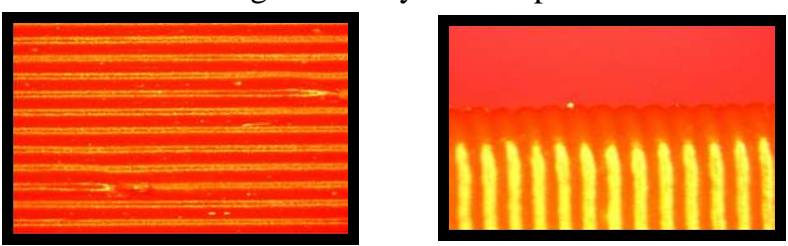

$27 \mathrm{kHz}$
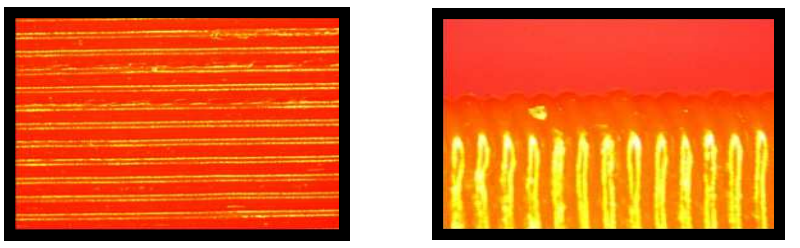

$40 \mathrm{kHz}$
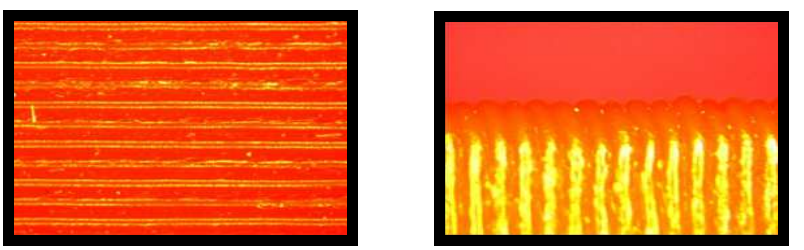

$50 \mathrm{kHz}$

Fig. 4a 1 piezoelectrics

The discussion of the results begins with analyzing the graph of surface roughness against number of piezo mounted on UP Plus 2 3D FDM printer. The interesting part of this data is that the FDM process which assisted by using 1 piezo with various frequencies indicate that by applying $50 \mathrm{kHz}$ of ultrasonic frequency, the surface roughness is smoother compared to 40 and $27 \mathrm{kHz}$ of frequency which generates a reading of 13.66, 13.70 and 14.03. Conversely, the data obtain when assisting with multiple number of piezo is slightly different. By using 4 piezo and a constant amount of frequency supplied, the result shows increasing value of quality surface roughness with a value of $13.82,13.88$ and 13.97 for each frequency. However, from this data, we can see that with 2 and 3 
piezo, the result shows the high value of surface roughness compared with 1 and 4 number of piezo.
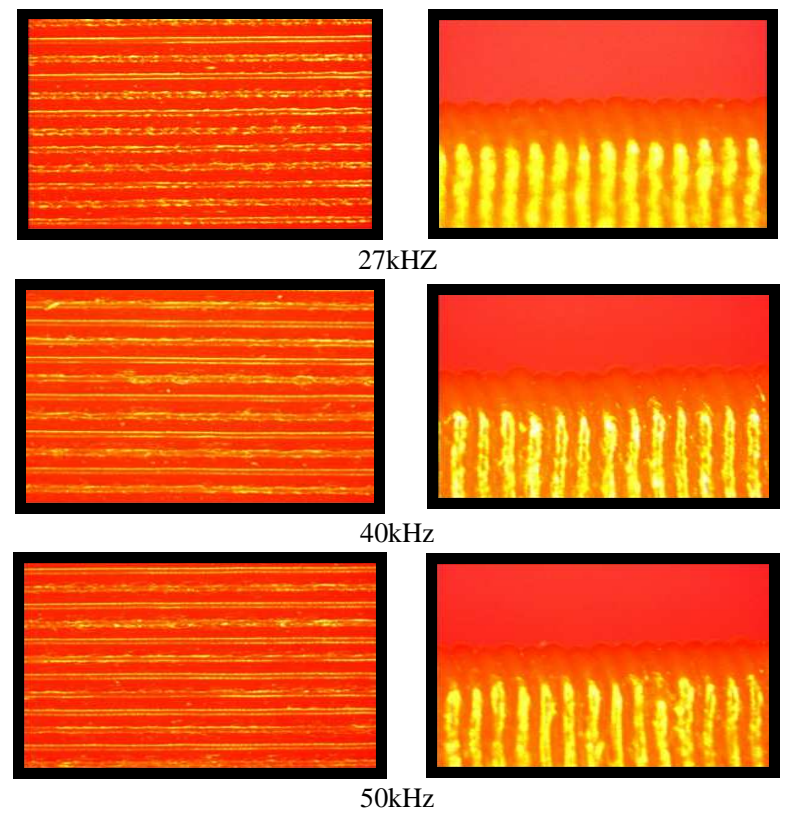

Fig. 4b 2 piezoelectrics
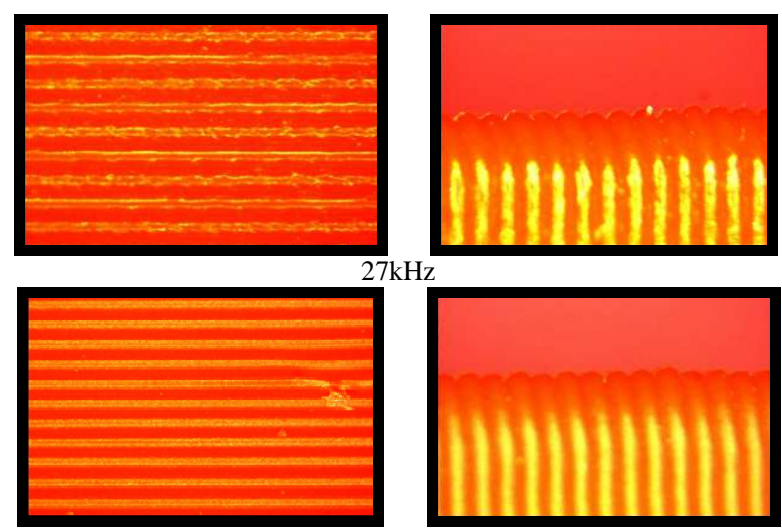

$40 \mathrm{kHz}$

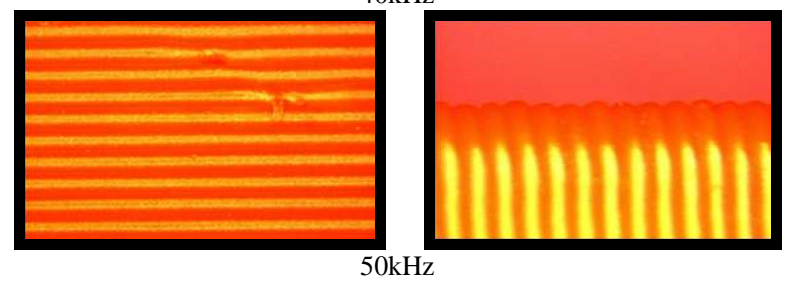

Fig. 4c 3 piezoelectrics

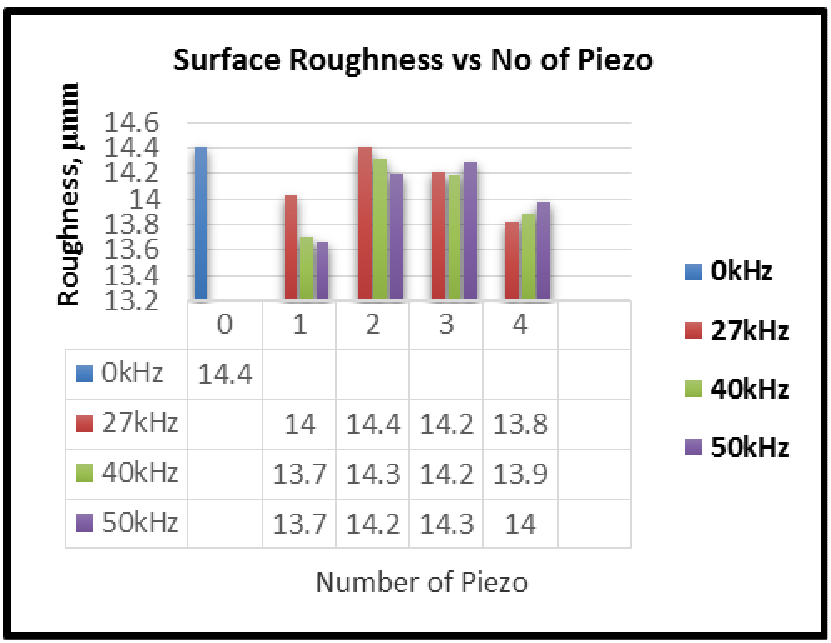

Fig. 5 Graph surface roughness versus number of piezo

\section{CONCLUSIONS}

The present study was designed to determine the effect of using multiple piezoelectric transducer on the FDM machine to enhance surface quality by considering the process parameter of layer thickness, frequency and fill of surface. Based on the previous research, it proves that the ultrasonic vibration can reduce the staircase effect during the printing process [26]. Thus, ultrasonic was able to make an improvement on the quality of the surface finish of the sample printed with the appropriate frequency applied.

However, it depends on the number and position of piezoelectric mounted on the 3D printer. The result shows that 1 piezo with $50 \mathrm{kHz}$ applied to the FDM hotbed platform printed the outstanding surface finish. The finding is consistent with the findings of past studies. By assisting piezoelectric vibration, the road width and layer thickness were successfully reduced from $0.09 \mathrm{~mm}$ to $0.05 \mathrm{~mm}$ due to compression occurred on the printed sample while vibration start to transmit and make contact with it. In addition, it showed that piezoelectric transducer could also minimize the defected layer thickness [26].

This research and the data obtain from the experiment have possibilities to be applied in several other AM systems such as SLA, SLS and Electron Beam Modeling technology. Thus, results will likely benefit in the future for the purpose of product design and development and reducing of manual process of hazardous and expensive post processing process which is time consuming. Furthermore, the findings of this experiment could likely potential to be applied in industries such as automotive, consumer product, medical, sports, etc. in creating prototypes or custom-made actual product or part. Further research, it is recommended to study its consistency of surface finish produced by printing a complex part using geometries that have curved surface and with a different degree of angles. On top of that, it is suggested that the investigation of the use of other material such as polyactic acid (PLA) or composite material should be conducted and the sample should be printed more than 1 sample for each frequency. 


\section{ACKNOWLEDGMENT}

The authors are grateful to all those who have assisted directly or indirectly to complete this study at the Universiti Teknikal Malaysia Melaka. The authors would like to thank the Ministry of Higher Education Malaysia for awarding the Fundamental Research Grant Scheme (FRGS) grant number FRGS/1/2015/TK03/FKP/02/F00282

\section{REFERENCES}

[1] D. T. Pham and R. S. Gault, "A comparison of rapid prototyping technologies," International Journal of Machine Tools and Manufacture, vol. 38, pp. 1257-1287, Oct. 1998.

[2] S. Choi and S. Samavedam, "Modelling and optimisation of rapid prototyping," Computers in Industry, vol. 47, pp. 39-53, Jan. 2002.

[3] F. Le Bourhis, O. Kerbrat, L. Dembinski, J.-Y. Hascoet and P. Mognol, "Predictive model for environmental assessment in additive manufacturing process," Procedia CIRP, vol. 15, pp. 26-31, Dec. 2014.

[4] I. Gibson, D. W. Rosen and B. Stucker, "Additive Manufacturing Technologies: 3D Printing, Rapid Prototyping, and Direct Digital Manufacturing, New York, USA: Springer, 2010.

[5] B. N. Panda, M. V. A. R. Bahubalendruni and B. B. Biswal, "Comparative evaluation of optimization algorithms at training of genetic programming for tensile strength prediction of FDM processed part," Procedia Materials Science, vol. 5, pp. 2250-2257, Dec. 2014.

[6] A. K. Sood, R. K. Ohdar and S. S. Mahapatra, "Parametric appraisal of fused deposition modelling process using the grey Taguchi method," Proceedings of the Institution of Mechanical Engineers, Part B: Journal of Engineering Manufacture, vol. 224, pp. 135-145, 2015.

[7] T. Tawakoli and B. A. Ã, "Influence of ultrasonic vibrations on dry grinding of soft steel," International Journal of Machine Tools and Manufacture, vol. 48, pp. 1585-1591, Nov. 2008.

[8] J. Pujana, A. Rivero, A. Celaya and L. N. López de Lacalle, "Analysis of ultrasonic-assisted drilling of Ti6Al4V," International Journal of Machine Tools and Manufacture, vol. 49, pp. 500-508, May 2009.

[9] P. Deepa, "Fused deposition modeling-A rapid prototyping technique for product cycle time reduction cost effectively in aerospace applications," IOSR Journal of Mechanical and Civil Engineering, vol. 2014, pp. 62-68, 2014.

[10] P. Jain and A. M. Kuthe, "Feasibility study of manufacturing using rapid prototyping: FDM approach," Procedia Engineering, vol. 63, pp. 4-11, Dec. 2013.

[11] G. C. Onwubolu and F. Rayegani, "Characterization and optimization of mechanical properties of ABS parts manufactured by the fused deposition modelling process," International Journal of Manufacturing Engineering, vol. 2014, p. 1-13, Nov. 2014.

[12] A. Kantaros and D. Karalekas, "Fiber Bragg grating based investigation of residual strains in ABS parts fabricated by fused deposition modeling process," Materials and Design, vol. 50, pp. 4450, Sep. 2013.

[13] M. Fischer and V. Schöppner, "Some investigation regarding the surface treatment of Ultem*9085 parts manufactured with fused deposition modeling," in Proc. 24th International SFF SymposiumAn Additive Manufacturing Conference, 2013, p. 805-815.

[14] A. K. Sood, R. K. Ohdar and S. S. Mahapatra, "Experimental investigation and empirical modelling of FDM process for compressive strength improvement," Journal of Advanced Research, vol. 3, pp. 81-90, Jan. 2012.

[15] R. I. Campbell, M. Martorelli and H. S. Lee, "Surface roughness visualisation for rapid prototyping models," Computer-Aided Design, vol. 34, pp. 717-725, Sep. 2002.
[16] A. Kumar, R. K. Ohdar and S. S. Mahapatra, "Improving dimensional accuracy of fused deposition modelling processed part using grey Taguchi method," Materials and Design, vol. 30, pp. 4243-4252, Dec. 2009.

[17] L. M. Galantucci, F. Lavecchia and G. Percoco, "Quantitative analysis of a chemical treatment to reduce roughness of parts fabricated using fused deposition modeling," CIRP AnnalsManufacturing Technology, vol. 59, pp. 247-250, Dec. 2010.

[18] D. Ahn, J. H. Kweon, S. Kwon, J. Song and S. Lee, "Representation of surface roughness in fused deposition modeling," Journal of Materials Processing Technology, vol. 209, pp. 5593-5600, Aug. 2009.

[19] B. Vasudevarao, D. P. Natarajan, M. Henderson A. Razdan, "Sensitivity of RP surface finish to process parameter variation," in Proc. SFF'00, 2000, p. 251-258.

[20] W. Tianming and X. Juntong, "Prototype surface micro-precision in fused deposition modeling process," Chinese Journal of Mechanical Engineering, vol. 20, pp.100-106, 2007.

[21] V. Tiwary, P. Arunkumar, A. S. Deshpande and V. Khorate, "Studying the effect of chemical treatment and fused deposition modelling process parameters on surface roughness to make acrylonitrile butadiene styrene patterns for investment casting process," International Journal of Rapid Manufacturing, vol. 5, pp. 276-288, 2015.

[22] A. Armillotta, "Assessment of surface quality on textured FDM prototypes," Rapid Prototyping Journal, vol. 12, pp. 35-41, Jan. 2006.

[23] L. M. Galantucci, F. Lavecchia and G. Percoco, "Experimental study aiming to enhance the surface finish of fused deposition modeled parts," CIRP Annals-Manufacturing Technology, vol. 58, pp. 189192, Dec. 2009.

[24] D. E. Brehl and T. A. Dow, "Review of vibration-assisted machining," Precision Engineering, vol. 32, pp. 153-172, Jul. 2008.

[25] B. Azarhoushang and J. Ã. Akbari, "Ultrasonic-assisted drilling of Inconel 738-LC," International Journal of Machine Tools and Manufacture, vol. 47, pp. 1027-1033, Jun. 2007.

[26] Y. S. Liao, Y. C. Chen and H. M. Lin, "Feasibility study of the ultrasonic vibration assisted drilling of Inconel superalloy," International Journal of Machine Tools and Manufacture, vol. 47, pp. 1988-1996, Oct. 2007.

[27] J. J. Yang, H. Zhang, X. Z. Deng and B. Y. Wei, "Ultrasonic lapping of hypoid gear: System design and experiments," Mechanism and Machine Theory, vol. 65, pp. 71-78, Jul. 2013.

[28] W. J. Kim, F. Lu, S. H. Cho, J. K. Park and M. G. Lee, "Design and optimization of ultrasonic vibration mechanism using PZT for precision laser machining," Physics Procedia, vol. 19, pp. 258-264, Dec. 2011.

[29] M. Nad, "Ultrasonic horn design for ultrasonic machining technologies," Applied and Computational Mechanics, vol. 4, pp. 7988, Mar. 2010.

[30] S. Maidin, M. K. Muhamad and E. Pei, "Feasibility study of ultrasonic frequency application on FDM to improve parts surface finish,” Jurnal Teknologi, vol. 77, pp. 27-35, Dec. 2015.

[31] Y. Gao, R. Sun and J. Leopold, J, "Analysis of cutting stability in vibration assisted machining using analytical predictive force model," Procedia CIRP, vol. 31, pp. 515-520, Dec. 2015.

[32] M. B. Mawale, A. M. Kuthe and S. W. Dahake, "Additive layered manufacturing: State-of-the-art applications in product innovation," Concurrent Engineering, pp. -19, Jan. 2016.

[33] S. S. Li, Y. B. Wu and M. Nomura, "Fundamental investigation of ultrasonic assisted surface grinding of Inconel 718," Advanced Materials Research, vol. 1136, pp. 365-370, 2016.

[34] R. Ibrahim, N. H. Rafai, E. A. Rahim, C. Kai and D. Hui, "A performance of 2 dimensional ultrasonic vibration assisted milling in cutting force reduction, on aluminium AL6061," in Proc. IRIS'15, 2015, p. $1-5$. 